\title{
Surgical Repair of Ankyloblepharon in Mallards (Anas platyrhynchos)
}

\author{
RenÉE SCHOtT, BS \\ Four LAKES Wildlife CENTER \\ University of Wisconsin-Madison School of Veterinary Medicine \\ MADISON, WisCONSIN
}

\begin{abstract}
Congenital and acquired ankyloblepharon (complete fusion of the upper and lower eyelids) have been documented in birds (Buyukmihci et al 1990; Pinard et al 2006). Surgical repair of ankyloblepharon in cockatiels (Nymphicus spp.) has been reported as unsuccessful in four cases of presumed congenital origin and successful in one case of acquired ankyloblepharon (Buyukmihci et al 1990; Pinard et al 2006). Two mallards (Anas platyrhynchos) with ankyloblepharon were treated with surgical reconstruction of the eyelids to restore bilateral vision. The first mallard presented as a one-week old orphan. Physical exam findings were normal except for a small amount of mucopurulent discharge from the left eye. Surgical reconstruction of the eyelids was performed successfully, with signs of wound contracture two weeks post-operatively, but no further closure at the three-week recheck. A full thickness corneal scar, anterior synechia, and small cataract were noted during surgery that suggest trauma as a cause for the ankyloblepharon. The second mallard presented with ankyloblepharon of unknown etiology. The first surgical reconstruction was unsuccessful, however a second attempt, using a more rigorous surgical eyelid excision, was successful with no further closure of the palpebral fissure at follow-up two months later. Moderate pododermatitis was successfully treated using systemic antibiotics and Collasate ${ }^{\text {TM }}$ (PRN Pharmacal, Pensacola, FL) regimen.
\end{abstract}

Keywords: ankyloblepharon, eyelids, avian, mallard, Anas platyrhynchos, ocular trauma, pododermatitis, Collasate ${ }^{\mathrm{TM}}$

\section{INTRODUCTION}

Ankyloblepharon is defined as adhesion of the ciliary margins of the upper and lower eyelids (Dordlund 2004). Ankyloblepharon can be partial or complete, and acquired or congenital. Acquired partial and complete ankyloblepharon can be a consequence of ocular trauma, photosensitization, feline herpesvirus, and ophthalmia neonatorum (newborn bacterial conjunctivitis [Although the term ankyloblepharon is used for a wide variety of clinical presentations in these reported cases.]) (Egyed et al 1975; Spiess 1985; Gelatt 2007). Acquired ankyloblepharon in humans has also been reported as part of Stevens-Johnson syndrome, which is an immune-mediated hypersensitivity reaction in

Renée Schott is a fourth-year veterinary student at the University of Wisconsin-Madison. After school, she hopes to specialize in wildlife and/or avian medicine. the skin and mucosal membranes causing sloughing of the epidermis and subsequent formation of scar tissue (Nordlund et al 2004). Delayed eyelid opening in neonatal animals is termed congenital ankyloblepharon, whereas cryptophthalmos is a developmental anomaly in formation of the eyelids with a complete absence of lid margins (Dordlund 2004; Gelatt 2007), and is often associated with other developmental eye anomalies (Koenigsberg 1989). In one case of acquired ankyloblepharon in a cockatiel, surgical reconstruction of the eyelids was successful (Pinard et al 2006). Other attempts to surgically reconstruct eyelids of cockatiels with fused palpebral margins have been unsuccessful (Buyukmihci et al 1990).

Two wild mallards with ankyloblepharon underwent reconstructive surgery to restore bilateral vision to enable release back into the wild. Some wildlife rehabilitators have policies of not releasing one-eyed animals as they would be less able to avoid predators and hunt for food; these animals are euthanized. Other wildlife rehabilitators routinely release oneeyed prey birds (Erica Miller, DVM, Tri-State Bird Rescue and Research, Inc., Newark, DE, personal communication). The first mallard underwent one surgery and while showing mild wound contracture two weeks post-operatively, no narrowing of the palpebral fissure was observed during the following week. After two surgeries, the second mallard recovered successfully with eyelids showing no fusion two months after the second surgery.

\section{MALLARD ANKYLOBLEPHARON CASES}

Case One. The first mallard presented as a oneweek old orphan to a local wildlife rehabilitation center that rehabilitates orphaned birds for release back into the wild. Physical exam findings were normal 


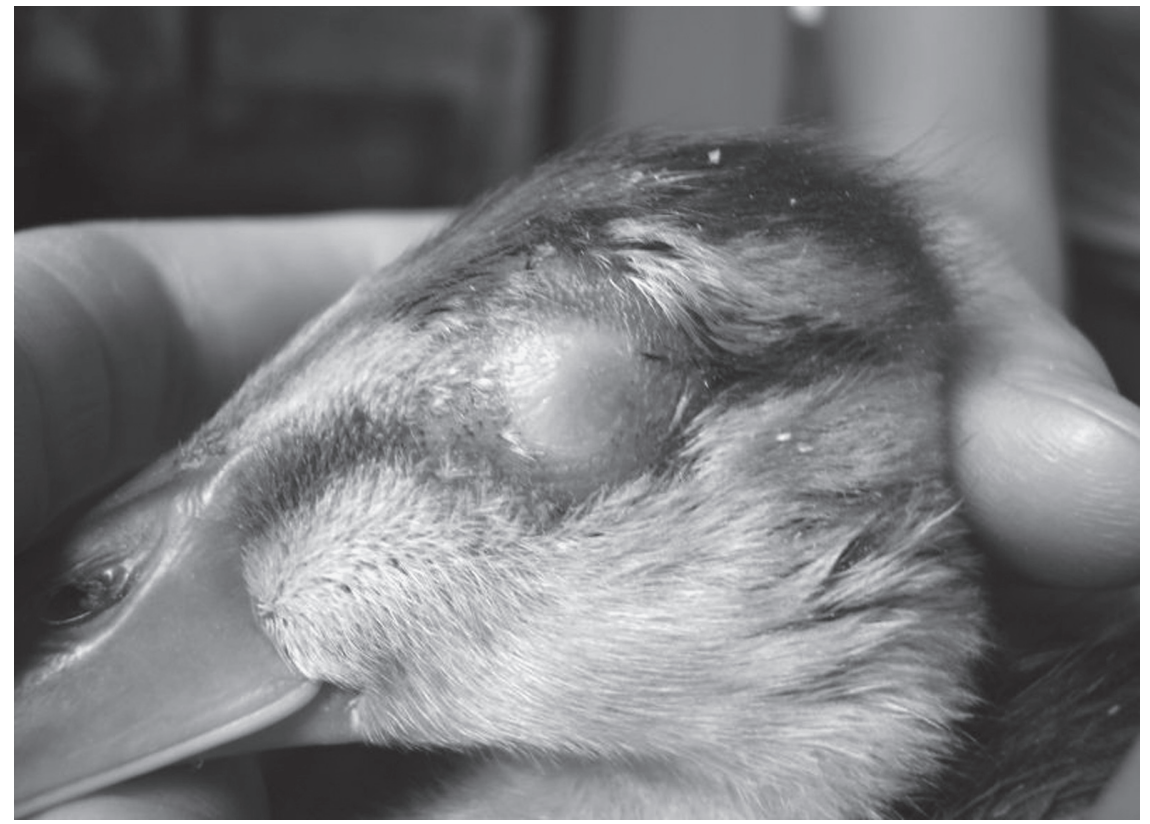

Figure 1. Case one mallard at three weeks old with complete ankyloblepharon of left eye.

mallard was recaptured on a local lake after one week. The mallard's body condition had deteriorated from a 4 to 3 on the body condition scale (where 1 is emaciated and 5 is obese), and it had developed a limp in the right leg. A soft tissue swelling surrounding the right tarsometarsal joint without evidence of bony involvement was seen radiographically. Differentials included cellulitis, hematoma, and edema. Mild pododermatitis had also developed on both feet. The skin around the affected left eye had taken on a greenish tint; however, pain, swelling, and discharge were absent. No other changes were observed.

Surgical reconstruction of the eyelids was performed when the except for a small amount of mucopurulent discharge from the left eye that was empirically treated with triple antibiotic eye ointment (Bacitracin, Neomycin, Polymyxin B) twice daily. Progressive narrowing of the palpebral fissure was noted, resulting in complete ankyloblepharon within two weeks. No palpebral fissure could be found at four weeks of age (Figure 1). The nictitans could be seen sweeping across the eye underneath the fused palpebrae. Absence of orbicularis oculi muscle contractions was noted after sensory stimulation of the eyelid with a 25-gauge needle. At six weeks old, the mallard was accidentally released with surrogate clutch mates. The

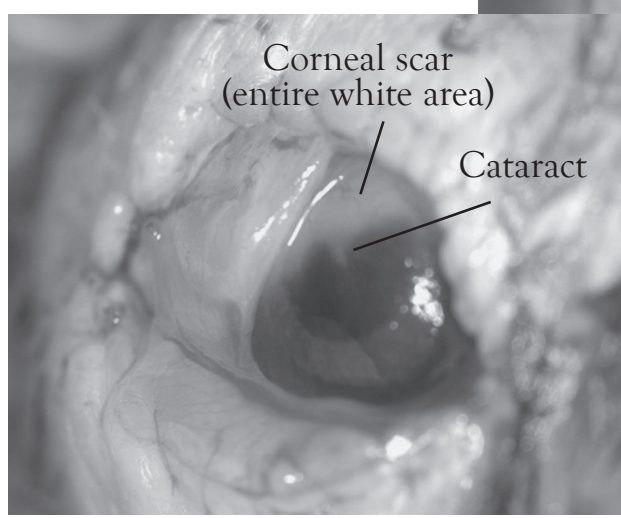

Figure 2a. Figure 2 detail; note corneal scar and cataract. mallard was approximately eight weeks old. General anesthesia was induced using isoflurane via a facemask. Torbugesic ${ }^{\circledR}$ (butorphanol, $1 \mathrm{mg} / \mathrm{kg}$, Fort Dodge, Madison, NJ) was given intramuscularly immediately

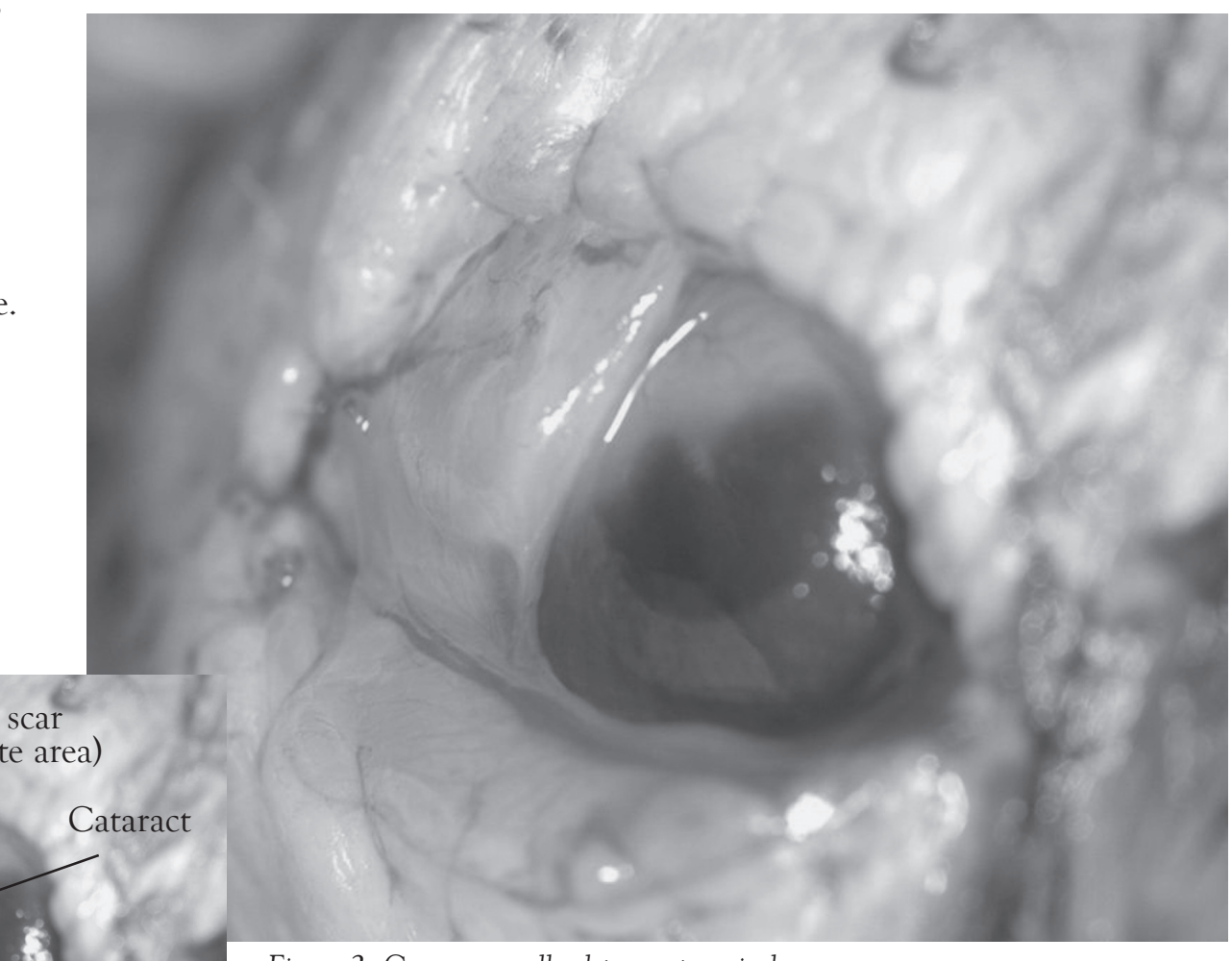

Figure 2. Case one mallard post-operatively.

after induction for pain management. The mallard was intubated (3.5 mm endotracheal tube) and maintained on 2 to 3 percent isoflurane throughout surgery. The surgical site was prepared by plucking the feathers around the eye and washing with irrigat- 
ing eyewash solution (Major Eye Wash ${ }^{\circledR}$, Major Pharmaceuticals, Livonia, MI) and a dilute betadine solution (1.25\% povidone iodine). Magnifying loupes (Carl Zeiss

Meditec, Inc., Prism Loupes, 4.5x magnification) were used for appropriate magnification during surgery. The length of the right (normal) palpebral fissure was measured to be $11 \mathrm{~mm}$ (0.4 in). A skin incision of equal length was made on the affected left side. Unexpected findings discovered intraoperatively included significant conjunctival adhesions and fibrosis, a full thickness corneal scar, anterior synechia, and a small anteronasal cataract lining up with the corneal scar (Figures 2 and 2a). The retina and pecten were visible and appeared normal; the eye appeared quiet and not inflamed. Some of the fibrous and/or membranous conjunctival adhesions were broken down; however, the third eyelid was not completely liberated. Lack of orbicularis oculi contraction pre-operatively suggested an absence of eyelid function, necessitating preservation of nictitans movement over the entire cornea. Completely liberating the third eyelid could have compromised function leading to ocular complications. The eyelids were reconstructed by apposing and suturing the conjunctival mucosa to the skin margin using 9-0 vicryl in a simple continuous pattern (Figure 3). Post-operative medication included one drop of neomycin/polymyxin/ dexamethasone ophthalmic suspension ( $0.1 \%$ dexamethasone) twice daily for one week, then once daily the following week, oral Metacam ${ }^{\circledR}$ (meloxicam, $0.1 \mathrm{mg} / \mathrm{kg}$, Merial, Duluth, GA) once daily for two weeks, and oral trimethoprim/ sulfamethoxazole (TMS) once daily for two weeks $(60 \mathrm{mg} / \mathrm{kg}, 40 \mathrm{mg}$ sulfa/mL, and $8 \mathrm{mg}$ trimethoprim/ $\mathrm{mL}$ ). TMS was necessary for treatment of the pododermatitis that had progressed from mild to moderate.

The pododermatitis was also treated with Collasate ${ }^{\mathrm{TM}}$ gel once daily. After thoroughly washing and drying the feet, a thin layer of Collasate ${ }^{\mathrm{TM}}$ was applied and allowed to dry.

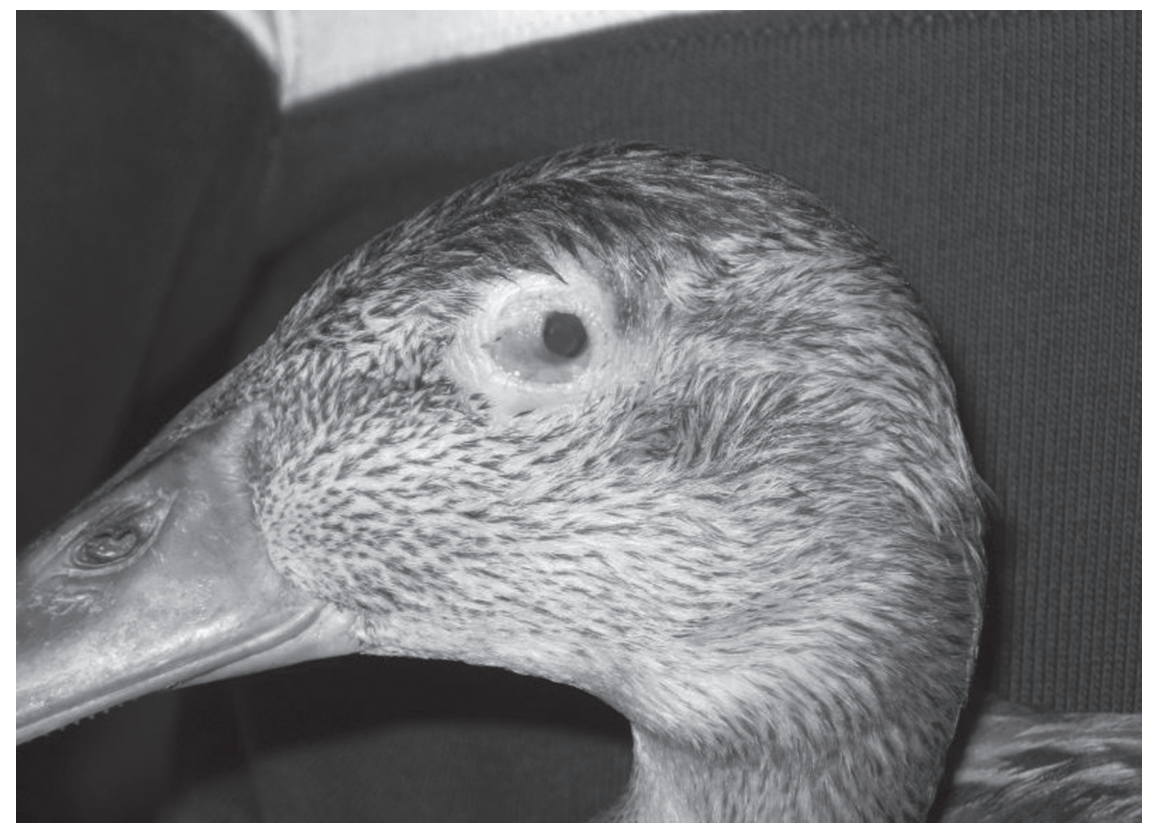

Figure 4. Case two mallard pre-operatively.

sions and small nasal cataract. The pododermatitis was nearly healed at the time of release. palpebral fissure showed mild shortening ( $9 \mathrm{~mm}[0.35$ in]). The pododermatitis had worsened after a significant initial improvement. The Collasate ${ }^{\mathrm{TM}}$ regimen that had been stopped four days previously was reinstated. At the three-week recheck, the palpebral fissure had not closed any further (Figure 4). A menace response was present despite nasal conjunctival adhe-

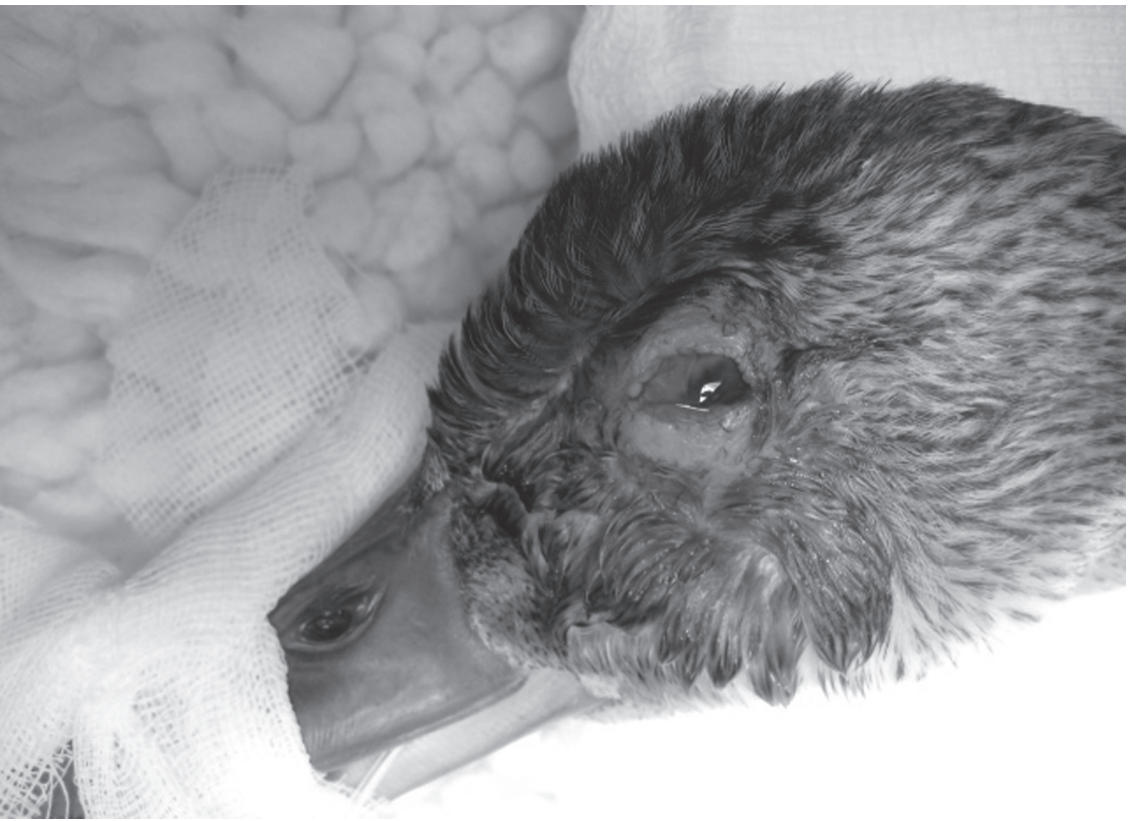

thee weeks post-operatively. 


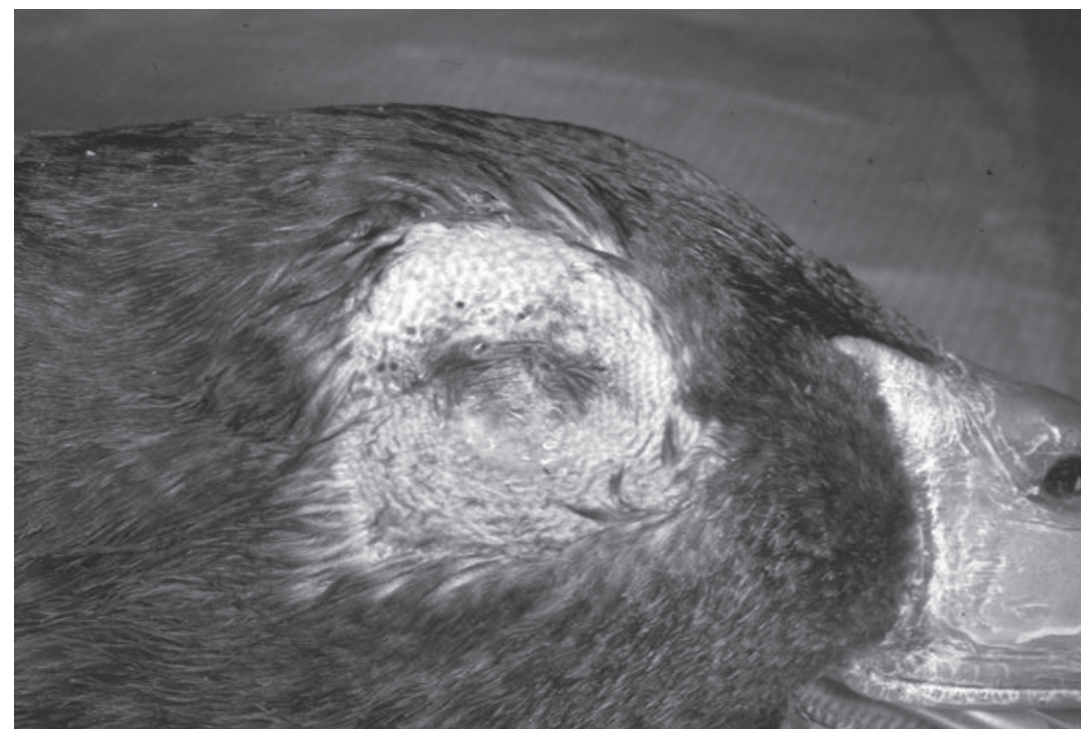

Figure 5. Case two mallard two weeks after first surgery.

Due to concerns regarding stress and season (Autumn), the mallard was released 25 days post-operatively near a lake that does not completely freeze over and where waterfowl are routinely fed all year.

Case Two. After being notified of a mallard's eye abnormality, a rehabilitator caught and subsequently brought the bird to the ophthalmologist for further evaluation and treatment. The juvenile female mallard presented to the ophthalmologist with right-sided ankyloblepharon (Figure 5). All other physical exam findings were normal.

The first surgical reconstruction was similar to that of the case one mallard previously described, although no ocular lesions were encountered intraoperatively. Two weeks postoperatively, the palpebral fissure had closed considerably (Figure 6). The second surgery involved a more rigorous excision of eyelid skin. The length of the palpebral fissure on the normal side was again measured together with the vertical diameter of the cornea (lower to upper limbus). These measurements were used to draw an ellipse on the skin of the affected side, such that the horizontal length of the ellipse equaled the palpebral fissure length of the normal eye and the vertical height of the ellipse equaled the vertical corneal diameter on the normal side. The ellipse was excised and the conjunctival mucosa was sutured to the skin margin. The postoperative care after both surgeries was similar to the post-operative care in case one. The palpebral fissure remained open with no signs of shortening after two months of follow-up.

\section{Discussion}

Case One. From the accidental release, the mallard sustained a soft tissue swelling of the left hind limb, had lost weight, and presented in a weakened state. This influenced the decision to take this mallard to surgery to increase the chance of survival. The unexpected findings of conjunctival adhesions and fibrosis, a full thickness corneal scar, anterior synechia, and a small anteronasal cataract lining up with the corneal scar suggested trauma and secondary infection as a possible cause of the ankyloblepharon; fibrosis as a result of wound healing has been implicated in the literature as a possible cause for ankyloblepharon (Egyed et al 1975, Spiess 1985, Pinard et al 2006). Buyukmihci et al (1990) described that post-operative narrowing of the

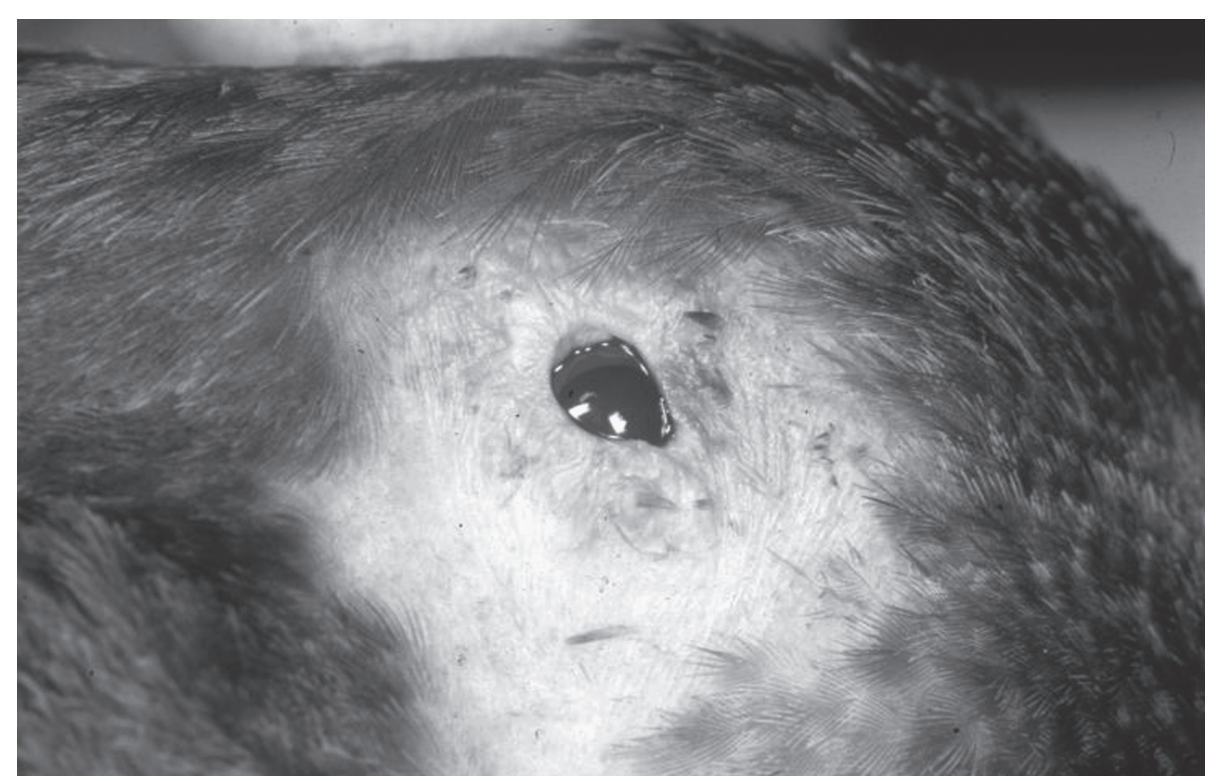

Figure 6. Case two mallard two months after second surgery.

palpebral fissures occurred within 10 days and complete closure was seen after two weeks to three months post-operatively. Ideally, a longer post-operative observation period would have allowed sufficient time for follow-up. However, this mallard was particularly stressed in captivity; it would only gain weight once accustomed to a daily routine, and would stop gaining weight for several days after a stressful situation 
(i.e., ophthalmic exam). This, in addition to imminent cold weather, drove the decision to release the mallard as soon as possible. Prolonged stress is detrimental to the health of animals, and frequently causes death in hospitalized wildlife patients (Fowler 1996; Macleod and Dragoumis 2000; Myers 2006). Stress in this mallard was evidenced by lack of appropriate, consistent weight gain, and abnormal behavior such as pacing. Keeping this mallard in captivity longer would not have been in its best interest. Long term success of this surgical technique cannot be determined definitely due to the short post-operative observation period. However, lack of palpebral closure in the last week of post-operative observation suggests the possibility that surgery was successful. If this surgery was successful, it is not known why; the author speculates it may be related to the type of ankyloblepharon (acquired) that has been documented as repairable (Pinard et al 2006), as opposed to a congenital ankyloblepharon in which surgery has been documented to fail (Buyukmihci et al 1990).

Case Two. Since this mallard was admitted with ankyloblepharon, it is unknown whether the condition was of acquired or congenital origin. A larger, elliptical excision was feasible in this case because the nictitating membrane was intact. It is speculated that the larger, more aggressive surgical excision separated the wound edges more and facilitated the lack of palpebral closure in the second attempt.

Surgical Results. Surgical correction of ankyloblepharon in birds has been largely unsuccessful; however, most of those cases were presumed to be of congenital origin (Buyukmihci et al 1990). One report showed long-term success in traumatically acquired ankyloblepharon (Pinard et al 2006). Presented in this paper are the cases of two mallards, one with an acquired and one with possibly congenital ankyloblepharon. Both cases were managed surgically with a good short and middle- to long-term outcome respectively. An interesting side note is that the case one mallard with acquired ankyloblepharon demonstrated no behavioral evidence of orbicularis oculi function. This is a feature characteristic of the abnormal eyelid development associated with cryptophthalmos. Taken together, these findings suggest that the lack of behavioral evidence of orbicularis oculi function is not pathognomonic for a congenital defect.
Literature CITED

Buyukmihci, N., C. Murphy, J. Paul-Murphy, D. Hacker, L. Laratta, and D. Brooks. 1990. Eyelid malformation in four cockatiels. Journal of American Veterinary Medical Association. 196(9): 14.90-14.92.

Dorlund Illustrated Medical Dictionary. Merck Source. c2004. Available from: <www.mercksource.com>.

Egyed, M., D. Dausch, and W. Wegner. 1975. Chronic lesions in geese photosensitized by Ammi majus. Avian Disease. 19(4):822-826.

Fowler, M. 1996. An overview of wildlife husbandry and diseases in captivity. Revue scientifique et technique de l'Office international des Epizooties. 15(1):15-22.

Gelatt, K. 2007. Veterinary Ophthalmology, 4th edition. Blackwell Publishing: Ames, IA.

Koenigsberg, R. 1989. Churchill's Medical Dictionary, 1st edition. Churchill Livingstone Inc: New York, NY.

Macleod, A., and P. Dragoumis. 2000. The Exotics Dilemma: A Case Study in Progress, Part 4: The Stress of Captivity. Journal of Wildlife Rehabilitation. 23(4):13-17.

Myers, D. 2006. Common Procedures and Concerns with Wildlife. Veterinary Clinics of North America: Exotics Animal Practice. 9:437-460.

Nordlund, M., E. Holland, and R. Kersten. 2004. Ocular Surface Reconstruction in a Patient With Complete Ankyloblepharon Resulting from Stevens-Johnson Syndrome. Archives of Ophthalmology. 122:934-935.

Pinard, C., G. Fitzgerald, and M. Desmarchelier. 2006. Surgical Repair of Acquired Ankyloblepharon in a Cockatiel. Journal of Avian Medicine and Surgery. 20(4):253-257.

Spiess, V. 1985. Symblepharon, pseudopterygium and partial ankyloblepharon as sequelae of feline herpetic keratoconjunctivitis. Kleintier-Praxis. 30(3):149-157. (상 